\title{
INSECTOS POLINIZADORES EN SISTEMAS DE PRODUCCIÓN DE Theobroma cacao L. EN LA ZONA CENTRAL DEL LITORAL ECUATORIANO
}

\section{POLLINATORS INSECTS IN Theobroma cacao L. PRODUCTION SYSTEMS IN THE CENTRAL AREA OF THE ECUADORIAN LITTORAL}

\author{
Vivianna Armijos Vásquez ${ }^{1}$, Luz Cecilia García Cruzatty², Jessenia Castro Olaya ${ }^{2}$ y Malena Martínez ${ }^{3}$ \\ ${ }^{1}$ Facultad de Ciencias Pecuarias, Universidad Técnica Estatal de Quevedo, Los Ríos-Ecuador \\ ${ }^{2}$ Facultad de Ingeniería Agronómica, Universidad Técnica de Manabi, Manabi-Ecuador, cecilialuz29@hotmail.com,130105, \\ $+593981783548$ \\ ${ }^{3}$ Facultad de Ciencias Ambientales, Universidad Técnica Estatal de Quevedo, Los Ríos-Ecuador \\ *Autor de correspondencia: cecilialuz29@hotmail.com
}

Rec.: 01.10.2020. Acept.: 28.09.2020.

Publicado el 30 de diciembre de 2020

\begin{abstract}
Resumen
Tos polinizadores naturales cumplen un rol importante en la eficiencia reproductiva de Theobroma cacao. El objetivo de esta investigación fue determinar la cantidad y diversidad de insectos en $T$. cacao tipo Nacional, en sistema monocultivo y sistema agroforestal, con énfasis en los polinizadores. El estudio se realizó en finca experimental "La Represa” ubicada en el cantón Quevedo, provincia Los Ríos, Ecuador. Se colocaron trampas de emergencia y captura de insectos en los dos sistemas de cultivo. Además, se realizaron observaciones directas en las flores de $T$. cacao para registrar la presencia de insectos polinizadores. Los insectos capturados se identificaron con la ayuda de claves entomológicas, lupa, y estereomicroscopio. Se calculó el índice de dominancia y diversidad Simpson e índice de similitud de Jaccard. Los mayores porcentajes de insectos se obtuvieron del orden Hymenoptera (76.99\%), Coleoptera (10.21\%) y Diptera (7.95\%). El índice Simpson fue de 0.58 y 0.42 para dominancia y diversidad, respectivamente. La similitud según Jaccard fue del $24 \%$ de las familias de insectos en los sistemas de producción. Se registró una sola familia (Cecidomyiidae) como un posible polinizador en $T$. cacao en el área de estudio. Hay baja diversidad y alta dominancia de insectos para los sistemas de producción analizados. Uno de los factores climáticos que influye en la presencia de los insectos capturados en $T$. cacao es la humedad relativa, pues se encontró una relación directa positiva entre estas dos variables.
\end{abstract}

Palabras claves: Agroforestal, Cecidomyiidae, Claves entomológicas, Diversidad, Monocultivo

\begin{abstract}
$\mathrm{N}^{2}$ atural pollinators follow an important role in the reproductive efficiency of Theobroma cacao L. The objective of this research was to determine the quantity and diversity of insects in T. cacao National Type, in monoculture system and agroforestry system, with emphasis on pollinators. The study was carried out in the experimental farm "La Represa" located in the city Quevedo, province Los Ríos, Ecuador. Emergency traps and insect capture were placed in the two farming systems. In addition, direct observations were made on the flowers of $T$. cacao to record the presence of pollinating insects. The captured insects were identified with the help of entomological keys, magnifying glass, and stereomicroscope. The Simpson dominance and diversity index and Jaccard similarity index were calculated. The highest percentages of insects were obtained from the order Hymenoptera (76.99\%), Coleoptera (10.21\%) and Diptera (7.95\%). The Simpson index was calculated at 0.58 and 0.42 , and Jaccard's similarity was $24 \%$ among insect families found in both production systems. A single family (Cecidomyiidae) was recorded as a possible pollinator in T. cacao in the study area. There is low diversity and high dominance of insects for the production systems analyzed. One of the climatic factors that influences the presence of the insects captured in $T$. cacao is the relative humidity, since a positive direct relationship was found between these two variables.
\end{abstract}

Keywords: Agroforestry, Cecidomyiidae, Diversity, Entomological keys, Monoculture 


\section{Introducción}

E cuador es uno de los principales productores de cacao tipo "Nacional", aportando el 50\% de la oferta mundial de cacao fino o de aroma. Hay cerca de 100,000 unidades productivas con más de 400,000 hectáreas de este cultivo, en su gran mayoría en la región Litoral (Amores et al., 2009). Sin embargo, a pesar de la gran demanda de este producto, según los registros históricos, la especie ha sufrido cambios significativos en la producción, debido principalmente a factores climáticos y enfermedades (Jaimez et al., 2008; De almeida, 2012).

En el cultivo de T. cacao todas las variedades son estrictamente de polinización entomófila, debido al reducido tamaño y estructura floral. Está documentado que esta actividad la realizan principalmente insectos del género Forcipomyia (Diptera: Ceratopogonidae), considerados los más eficientes polinizadores de esta especie (Mavisoy et al., 2009).

En varios países productores de T. cacao, el patrón de floración, y lo que conlleva a la producción de flores, se ve directa o indirectamente influenciado por los factores climáticos del entorno (Alvim, 1984; Mohr y Schopfer, 1994). Sin embargo, se ha observado que períodos excesivamente secos o relativamente fríos pueden inhibir completamente la floración en las regiones donde persiste la variabilidad estacional de la precipitación y la temperatura (Alman, 1966; Asomaning et al., 1971). Una planta de T. cacao adulta puede producir miles de flores por año, a veces más de 50,000, de las cuales sólo una pequeña proporción es fertilizada (normalmente menos del 5\%) afectando directamente el potencial productivo de la especie (Alvim, 1984).

En Ecuador existen bajos rendimientos en el cultivo de T. cacao, puesto que existen limitantes que influyen directamente en la producción, siendo las plantaciones afectadas por diversos factores tales como: genéticos, ecológicos, fisiológicos, patógenos, y culturales (Noriega, 2012). No obstante, los agricultores e investigadores deducen que la bajas producciones de las huertas cacaoteras, pueden deberse precisamente a la poca presencia de insectos que contribuyen a la polinización (Córdoba et al., 2013). Teniendo en cuenta esta problemática se planteó esta investigación, con el objetivo de determinar la diversidad y abundancia de los insectos polinizadores de T. cacao tipo Nacional en sistema monocultivo y sistema agroforestal.

\section{Materiales y métodos}

\section{Ubicación de la zona de estudio}

El trabajo se llevó a cabo en la finca experimental "La Represa", propiedad de la Universidad Técnica Estatal de Quevedo (UTEQ), provincia Los Ríos, Ecuador. Su ubicación geográfica corresponde a $01^{\circ} 03^{\prime} 18^{\prime \prime}$ de latitud Sur y $79^{\circ} 25^{\prime} 24^{\prime \prime}$ de longitud Oeste, localizada en una zona clasificada como bosque húmedo-tropical a una altura de $73 \mathrm{msnm}$ con topografía ligeramente ondulada. Las principales características climáticas se muestran en el Cuadro 1.

Cuadro 1. Características climáticas anuales de la finca experimental "La Represa"

\begin{tabular}{lc}
\hline \multicolumn{1}{c}{ Parámetros } & Promedios \\
\hline Temperatura $\left({ }^{\circ} \mathrm{C}\right)$ & 25.47 \\
Humedad relativa $(\%)$ & 85.84 \\
Precipitación (mm) & 2223.78 \\
Heliofanía (horas/ luz /año) & 898.77 \\
Evaporación (\%) & 78.30 \\
Zona ecológica & Bh-T \\
\hline
\end{tabular}

Fuente: Estación Experimental Tropical Pichilingue (INIAP), 2015.

\section{Material vegetal}

El estudio se realizó en un ensayo multiclonal de T. cacao tipo Nacional, y Trinitario. Los clones de tipo Nacional utilizados fueron los siguientes: DICYT-C114, DICYT-C107, DICYT-C119, DICYT-C217, y DICYT-C186. Mientras que los Trinitarios fueron: CCN-51 y LR-35. También se estudió el clon EET544 (Sistema Agroforestal), establecido en asociación con especies forestales (Tectona grandis L., Triplaris cumingiana Fisch. \& Mey.). Los clones evaluados en el ensayo clonal están establecidos a una distancia de siembra de 3 × 3 metros (1111 plantas por hectárea), en un sistema de hilera simple con 10 rametos por clon.

\section{Captura e identificación de insectos}

Para conocer la diversidad de insectos de los clones en estudio, se colocaron recipientes plásticos con hojarascas de T. cacao y fruto maduro de Musa paradisiaca L. en descomposición, para la atracción y captura de los insectos (Bravo et al, 2011). En la tapa se realizó una abertura que se cubrió con tela mosquitero de color negro que impedía la entrada de luz, y permitía el paso de humedad y aire. Se adicionó un tubo plástico con agua azucarada para atraer a los insectos que emergieran y a los que se encontraran en la superficie del suelo. Además, se efectuó un registro de los visitantes que llegaron hasta las flores mediante observación directa utilizando lupas de mano, fotografías y la captura de algunos ejemplares para su identificación. El registro de visitantes florales se realizó por 13 días consecutivos (febrero 2016), en las horas de la mañana, entre las $8 \mathrm{~h} 00$ y $12 \mathrm{~h} 30$, tomando 
como referencia tres rametos por clon, en siete clones del sistema de monocultivo y un clon del sistema agroforestal, donde se registró los insectos que llegaban a la flor y el tiempo de permanencia en esta.

Los insectos obtenidos para este ensayo fueron capturados entre los meses de octubre y marzo del 2016 (un total de 24 semanas), las trampas de insectos se retiraron tres veces a la semana; estos fueron conservados en alcohol (70\%) para luego ser trasladados al Laboratorio de Entomología de la Estación Experimental Tropical Pichilingue, INIAP, para su posterior clasificación e identificación, utilizando claves entomológicas, lupa eléctrica y microscopio binocular. La abundancia de insectos polinizadores y otros insectos, se estudió mediante el conteo de los insectos recolectados.

\section{Análisis estadístico}

Se evaluaron dos sistemas de producción (monocultivo y agroforestal) y tres unidades experimentales por cada uno. Para conocer la diversidad y dominancia de insectos en el sistema monocultivo y sistema agroforestal se utilizó el índice de Simpson, empleando la fórmula 1 :

$$
D=\frac{\sum_{\mathrm{I}=1}^{\mathrm{S}} \mathrm{n}_{\mathrm{i}}\left(\mathrm{n}_{\mathrm{i}}-1\right)}{\mathrm{N}(\mathrm{N}-1)}
$$

Se contabilizaron todos los insectos capturados, independientemente de la familia, y posteriormente se calculó la representación respectiva de cada familia. Para conocer la similitud que existió en los dos sistemas de producción se utilizó el índice de similitud de Jaccard, el cual se expresa en la fórmula 2:

$$
I_{j}=\frac{c}{\mathrm{a}+\mathrm{b}-\mathrm{c}}
$$

Para conocer la relación entre la cantidad de insectos y las variables climáticas se calculó el coeficiente de correlación de Pearson y se realizaron los gráficos de dispersión de puntos. Para realizar los gráficos y para el cálculo del índice de correlación se utilizó el programa Statistica 7.0.

\section{Resultados}

D urante el tiempo de evaluación, se capturó una diversidad de insectos representados por los órdenes Coleoptera, Diptera, Hemiptera, Hymenoptera, Ortoptera, Tysanoptera, obteniéndose una mayor cantidad de familias en el orden Coleoptera, y en menor cantidad de individuos del orden Ortoptera y Tysanoptera. Se identificó un total de 578 especímenes pertenecientes a 27 familias y 6 órdenes entre el sistema monocultivo y sistema agroforestal (Cuadro 2).

Los mayores promedios de insectos capturados fueron obtenidos en los meses de noviembre (27.34) y diciembre (26.82) y en menor porcentaje en el mes de marzo con un $9.69 \%$. El orden Hymenoptera fue el grupo más abundante, con un 76.99\%. El mayor número de ejemplares fueron capturados durante los meses de noviembre y diciembre, mientras que para el mes de marzo se reportaron pocas Hymenopteras (Cuadro 2).

El orden Coleoptera fue el segundo grupo con un $10.21 \%$, identificándose 10 familias, entre las que se destacan Curculionidae con 16 ejemplares en el mes de diciembre con un porcentaje a nivel de familia de 3.98\%, así mismo los Chrysomelidae, Dynastinae y Lucanidae se reportan en menor cantidad (0.17\%) (Cuadro 2 ).

Los insectos del orden Diptera constituyeron el tercer grupo más relevante en los meses de observación, con un $7.09 \%$, en el que destaca la familia Drosophilidae con un $2.94 \%$, seguido de la familia Cecidomidae con el $1.24 \%$. Estas especies son agalladoras, sin embargo, también son importantes polinizadores. Las Hemipteras, Ortopteras y Tysanopteras fueron los grupos de menor abundancia (Cuadro 2).

En general, el mayor porcentaje de insectos polinizadores se registró en el sistema de monocultivo con $3.63 \%$, mientras el $76.99 \%$ corresponde a otros insectos. Para el sistema agroforestal el porcentaje de insectos polinizadores fue de $0.17 \%$ y $19.20 \%$ representa a otros insectos capturados (Cuadro 3).

Los índices de dominancia y diversidad en el sistema agroforestal fueron de 0.58 y 0.42 respectivamente, mientras que en los clones de cacao la dominancia osciló entre 0.44 y 0.80 y la diversidad presentó valores entre 0.20 y 0.56 (Cuadro 4).

Se obtuvo mayor dominancia de insectos en los clones CCN-51 (0.80) y C-107 (0.70), así mismo, el clon que presentó menos dominancia de insectos fue el LR-35 (0.44); sin embargo, en este clon se encontró una mayor diversidad de individuos (0.56) (Cuadro 4).

Según el índice de similitud de Jaccard, hay un $24 \%$ de similitud en familias de insectos entre los sistemas de monocultivo y agroforestal, en el cual el sistema de monocultivo mostró mayor presencia de especies de insectos. En ambos sistemas de producción se encontraron especies de las familias: Scarabeidae, Curculionidae, Staphylinidae, Drosophilidae, Cecidomyiidae y Formicidae.

En el Cuadro 5 se detalla la diversidad de órdenes de insectos que visitan las flores de T. cacao, entre los cuales se identificaron cinco órdenes de insectos; entre ellas: Coleopteras, Hymenopteras, Dipteras y Hemipteras. Entre los dípteros identificados anteriormente como polinizadoras de T. cacao, se observó Cecidomyiidae. Se 
Cuadro 2. Insectos capturados durante seis meses en ensayo clonal de T. cacao en la finca experimental La Represa, Los Ríos- Ecuador, 2015-2016

\begin{tabular}{|c|c|c|c|c|c|c|c|c|c|c|}
\hline Orden & Familia & Condición & Oct. & Nov. & Dic. & Ene. & Feb. & Mar. & $\begin{array}{c}\% \text { de } \\
\text { insectos } \\
\text { por } \\
\text { familia }\end{array}$ & $\begin{array}{c}\% \text { de } \\
\text { insectos } \\
\text { por orden }\end{array}$ \\
\hline \multirow{4}{*}{ Hymenoptera } & (Formicidae) & $\begin{array}{c}\text { Viven en } \\
\text { simbiosis } \\
\text { (Hemiptera) }\end{array}$ & 29 & 123 & 104 & 52 & 53 & 36 & 68.69 & \multirow{4}{*}{76.99} \\
\hline & (Formicidae) & Visitantes & 21 & 1 & 1 & 1 & 0 & 6 & 5.19 & \\
\hline & (Formicidae) & Visitantes & 0 & 17 & 0 & 0 & 0 & 0 & 2.94 & \\
\hline & Vespidae & $\begin{array}{c}\text { Insecto } \\
\text { benéfico } \\
\text { (Depredador) }\end{array}$ & 0 & 0 & 1 & 0 & 0 & 0 & 0.17 & \\
\hline \multirow{10}{*}{ Coleoptera } & Scarabeidae & $\begin{array}{l}\text { Insecto plaga } \\
\text { (Defoliador) }\end{array}$ & 0 & 1 & 8 & 0 & 5 & 2 & 2.77 & \multirow{10}{*}{10.21} \\
\hline & Curculionidae & Insecto plaga & 0 & 1 & 16 & 4 & 1 & 1 & 3.98 & \\
\hline & Coccinelidae & $\begin{array}{l}\text { benéfico } \\
\text { (Controlador } \\
\text { biológico) }\end{array}$ & 0 & 1 & 1 & 0 & 0 & 0 & 0.35 & \\
\hline & Languriidae & $\begin{array}{l}\text { Insecto } \\
\text { depredador }\end{array}$ & 0 & 1 & 1 & 0 & 0 & 0 & 0.35 & \\
\hline & Hydroscaphidae & Insecto plaga & 1 & 2 & 1 & 0 & 0 & 0 & 0.69 & \\
\hline & Chrysomelidae & Insecto plaga & 0 & 1 & 0 & 0 & 0 & 0 & 0.17 & \\
\hline & Staphylinidae & $\begin{array}{l}\text { Insecto } \\
\text { depredador }\end{array}$ & 1 & 1 & 1 & 1 & 0 & 3 & 1.21 & \\
\hline & Bothrideridae & Insecto plaga & 1 & 0 & 1 & 0 & 0 & 0 & 0.35 & \\
\hline & Dynastinae & Insecto plaga & 0 & 0 & 1 & 0 & 0 & 0 & 0.17 & \\
\hline & Lucanidae & Insecto plaga & 0 & 0 & 0 & 1 & 0 & 0 & 0.17 & \\
\hline \multirow{5}{*}{ Diptera } & Drosophilidae & Visitante & 0 & 5 & 7 & 0 & 2 & 3 & 2.94 & \multirow{5}{*}{7.09} \\
\hline & Cecidomyiidae & $\begin{array}{l}\text { Posible } \\
\text { Polinizador }\end{array}$ & 0 & 1 & 1 & 0 & 3 & 2 & 1.21 & \\
\hline & Calliphoridae & Visitante & 0 & 2 & 5 & 0 & 0 & 0 & 1.21 & \\
\hline & Dolichopodidae & Visitante & 1 & 0 & 0 & 0 & 0 & 0 & 0.17 & \\
\hline & Faniidae & Visitante & 0 & 0 & 5 & 3 & 1 & 0 & 1.56 & \\
\hline \multirow{6}{*}{ Hemiptera } & Aphididae & Insecto plaga & 8 & 0 & 0 & 0 & 0 & 3 & 1.90 & \multirow{6}{*}{5.36} \\
\hline & Cicadellidae & Insecto plaga & 3 & 0 & 0 & 0 & 0 & 0 & 0.52 & \\
\hline & Delphacidae & Insecto plaga & 1 & 0 & 0 & 0 & 0 & 0 & 0.17 & \\
\hline & Pseudococcidae & Insecto plaga & 1 & 0 & 0 & 0 & 2 & 0 & 0.52 & \\
\hline & Lygacidae & Insecto plaga & 0 & 1 & 0 & 0 & 0 & 0 & 0.17 & \\
\hline & Miridae & Insecto plaga & 12 & 0 & 0 & 0 & 0 & 0 & 2.08 & \\
\hline Ortoptera & Grillydae & & 1 & 0 & 0 & 0 & 0 & 0 & 0.17 & 0.17 \\
\hline Tysanoptera & Triphidae & $\begin{array}{c}\text { Insecto } \\
\text { intermedio }\end{array}$ & 0 & 0 & 1 & 0 & 0 & 0 & 0.17 & 0.17 \\
\hline Total & & & 80 & 158 & 155 & 62 & 67 & 56 & & \multirow{2}{*}{100} \\
\hline$\%$ & & & 13.8 & 27.3 & 26.8 & 10.7 & 11.6 & 9.7 & 100 & \\
\hline
\end{tabular}


Cuadro 3. Promedio mensual de la abundancia de insectos capturados durante los seis meses en ensayos de T. cacao en monocultivo y sistema agroforestal de la finca experimental "La Represa", 2015-2016

\begin{tabular}{ccccccccccc}
\hline Sistemas & & Oct. & Nov. & Dic. & Ene. & Feb. & Mar. & Total & X & $\%$ \\
\hline \multirow{4}{*}{ Agroforestal } & Polinizadores & 0.00 & 0.00 & 0.00 & 0.00 & 0.33 & 0.00 & 0.33 & 0.06 & 0.17 \\
& Otros Insectos & 0.00 & 21.33 & 7.33 & 2.00 & 5.00 & 1.33 & 37.00 & 6.17 & 19.20 \\
\hline \multirow{2}{*}{ Monocultivo } & Polinizadores & 0.00 & 2.00 & 2.00 & 0.00 & 1.33 & 1.67 & 7.00 & 1.17 & 3.63 \\
& Otros Insectos & 26.67 & 29.00 & 42.33 & 18.67 & 16.00 & 15.67 & 148.33 & 24.72 & 76.99 \\
\hline
\end{tabular}

Cuadro 4. Índice de diversidad y dominancia de Simpson en insectos capturados en monocultivo y sistema agroforestal de T. cacao en la finca experimental La Represa, 2015-2016

\begin{tabular}{cccccccccc}
\hline Índice & CCN-51 & C-107 & C-109 & C-217 & C-114 & C-186 & LR-35 & $\begin{array}{c}\text { Sistema } \\
\text { agroforestal }\end{array}$ & Promedio \\
\hline Dominancia & 0.80 & 0.70 & 0.54 & 0.51 & 0.55 & 0.52 & 0.44 & 0.58 & 0.58 \\
\hline Diversidad & 0.20 & 0.30 & 0.46 & 0.49 & 0.45 & 0.48 & 0.56 & 0.42 & 0.42 \\
\hline
\end{tabular}

Cuadro 5. Visitantes florales de T. cacao en Monocultivo y Sistema agroforestal en la finca experimental La Represa, 2015- 2016

\begin{tabular}{|c|c|c|c|c|c|}
\hline $\begin{array}{l}\text { Comportamiento } \\
\text { de los visitadores }\end{array}$ & Orden & $\begin{array}{l}\mathrm{N}^{\mathrm{o}} \text { de } \\
\text { Insectos }\end{array}$ & $\begin{array}{l}\mathrm{N}^{\mathrm{o}} \text { de flores } \\
\text { visitadas por } \\
\text { insectos }\end{array}$ & Hora (am) & Observaciones \\
\hline \multirow{4}{*}{ Visitantes } & Coleoptera, & 8 & $1-2$ & $9 \mathrm{~h} 30-11 \mathrm{~h} 30$ & $\begin{array}{l}\text { Recorren la flor y se alimentan de } \\
\text { ella. }\end{array}$ \\
\hline & Hymenoptera & 6 & $1-3$ & $8 \mathrm{~h} 00-12 \mathrm{~h} 30$ & $\begin{array}{l}\text { Se alimentan de las excretas de } \\
\text { los áfidos que se encuentran en las } \\
\text { flores. }\end{array}$ \\
\hline & Diptera & 1 & $1-2$ & $9 \mathrm{~h} 00-11 \mathrm{~h} 00$ & $\begin{array}{l}\text { Vuelan por el árbol buscando flores } \\
\text { para posarse en los pétalos, sépalos, } \\
\text { brácteas y estaminoides, quedándose } \\
\text { quieta en un solo lugar. }\end{array}$ \\
\hline & Hemiptera & 1 & $1-2$ & $8 \mathrm{~h} 00-12 \mathrm{~h} 30$ & $\begin{array}{l}\text { Recorren toda la flor y algunos } \\
\text { insectos de este orden succionan } \\
\text { sabia }\end{array}$ \\
\hline Polinizadores & Diptera & 6 & $1-2$ & $9 \mathrm{~h} 00-11 \mathrm{~h} 00$ & $\begin{array}{l}\text { Vuelan por el árbol buscando flores } \\
\text { para posarse en los pétalos, sépalos, } \\
\text { brácteas y estaminoides, quedándose } \\
\text { quietos en un solo lugar. }\end{array}$ \\
\hline
\end{tabular}

encontró que, por lo general, las "mosquitas" visitaron las flores de T. cacao entre las $9 \mathrm{~h} 00$ y $11 \mathrm{~h} 00$ y solo se posaban en flores que no estuvieran con presencia de otros insectos. Los insectos que visitaban las flores del T. cacao con mayor frecuencia fueron de los órdenes Hymenopteras y Dipteras.

No se evidenció relación entre la temperatura, tanto mínima como máxima con la cantidad de insectos (Fig. 1). Pero sí existió correlación significativa $(r=0.52$; $p=0.000$ ) entre la humedad del ambiente y la cantidad de insectos encontrados, es decir que el $52 \%$ de la variabilidad en el número de insectos encontrados puede explicarse a través de la humedad relativa (Fig. 2). 


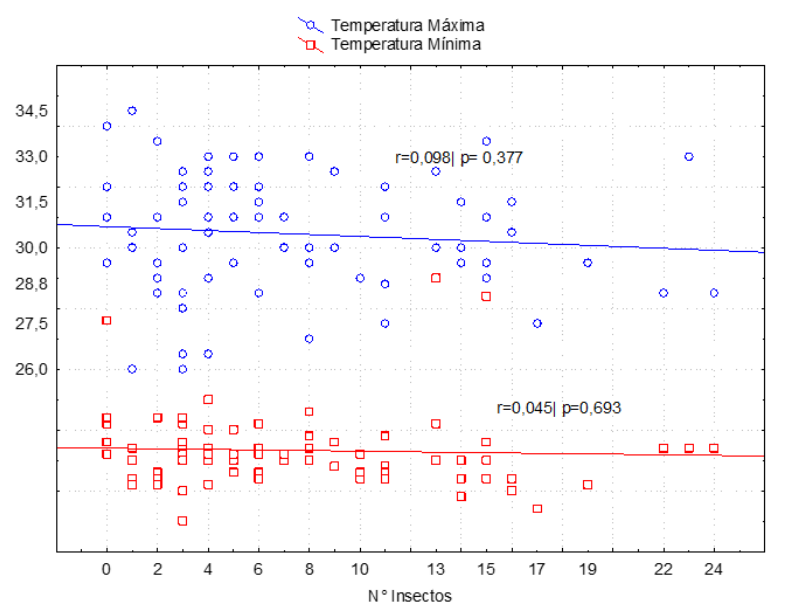

Figura 1. Correlación entre las temperaturas máxima y mínima y la presencia de insectos en ensayo clonal de $T$. cacao durante seis meses (octubre a marzo) en la finca La Represa, 2015-2016.

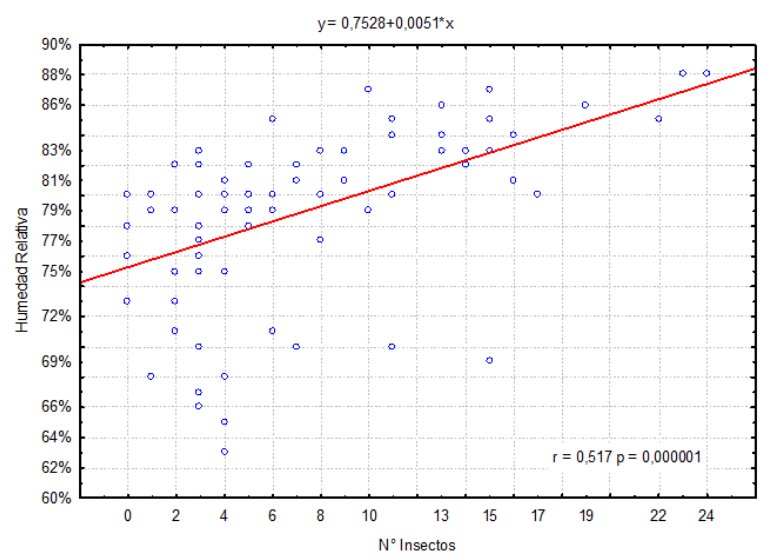

Figura 2. Correlación entre la humedad relativa y la presencia de insectos en ensayo clonal de $T$. cacao durante seis meses (octubre a marzo) en la finca La Represa, 2015-2016.

\section{Discusión}

$\mathrm{E}$ 1 tercer grupo más importante de insectos en el presente estudio corresponde al orden Diptera con un $7.09 \%$, que es inferior a lo obtenido por Bravo et al., (2011) quienes reportaron un 13.4\% de dípteros en un sistema agroforestal en T. cacao. Estos valores suelen variar dependiendo de la edad fisiológica del cultivo, evidenciándose con más frecuencia la presencia de insectos en plantaciones fisiológicamente productivas (Soria, 1979). No obstante, estos valores son similares a lo expuesto por Solano (2010), quien registró Hymenopteras en un 82.25\%, Coleopteras en un $8.03 \%$ y Dipteras $8.10 \%$. Según este autor, los grupos de insectos más representativos en los cacaotales ya sea en sistema de monocultivo o agroforestal son los tres grupos antes mencionados, siempre y cuando las características agroclimáticas del entorno presten las condiciones adecuadas para el equilibrio ecológico de estos insectos.

Por otra parte, se menciona que la abundancia total de polinizadores en relación al total de artrópodos en sistema agroforestal de T. cacao es alta (0.92\%) (Bravo et al., 2011). Valores que contrastan con los obtenidos en el presente estudio, donde se registra un $0.17 \%$ de posibles polinizadores del total de artrópodos capturados en sistema agroforestal. Sin embargo, se calculó un $3.0 \%$ en el sistema de monocultivo, los mismos que corresponden a la familia Cecidomyiidae. Soria et al., (1981) en un estudio realizado en Costa Rica, reportaron un $0.31 \%$ de artrópodos capturados pertenecientes a la familia Ceratopogonidae. En el presente estudio se obtuvo mayor proporción de insectos polinizadores pertenecientes a la familia Cecidomyiidae, en contraste a lo manifestado por Soria et al., (1981) estos insectos son los que visitaban con mayor frecuencia las flores de $T$. cacao. Así mismo, se menciona que la presencia de microdípteros (Forcipomyia spp), e insectos de la familia Cecidomyiidae ayudan a la deposición de masas de polen a los estigmas de las flores en T. cacao, actuando como polinizadores directos. Esto concuerda con Adjalo \& Oduro (2013) quienes describen que la mayoría de los visitantes habituales observados en las flores pertenecen a Hymenopteros y Dipteros, y no se incluyen polinizadores de la familia Ceratopogonidae.

A pesar de que Forcipomyia spp, solía ser considerado como el polinizador más importantes en T. cacao, con respecto a insectos de la familia Cecidomyiidae (Córdoba et al., 2013). Este último grupo deposita un mayor número de granos de polen por visita, lo cual lo convierte en un grupo muy importante de polinizadores efectivos. Cabe mencionar, que en esta investigación no se reportó Forcipomyia spp, no obstante, insectos de la familia Cecidomyiidae estuvieron presentes con escasa frecuencia. Según, Soria, (1979) la abundancia de polinizadores dípteros está directamente relacionada con la disponibilidad de agua en el suelo y la humedad relativa. En general, las poblaciones son mayores en periodos de precipitación y menores en periodos de sequía, lo que podría ser la causa por la cual no se registraron cantidades importantes de estos insectos.

La receptividad estigmática en clones de $T$. cacao inicia a partir de las 9h00 hasta las 11h00, extendiéndose muchas veces hasta las horas de la tarde (Mena, 2016), horarios que coinciden con la frecuencia de visita de dípteros (Cecidomyiidae) a flores de T. cacao. Por otra parte, Kaufmann (1973) afirma que la polinización en $T$. cacao la realizan insectos de la familia Cecidomyiidae en un 40 a $90 \%$, ya que estos insectos para realizar la deposiciones de sus huevos, lo hacen en las lígulas de las flores, trasportando directamente los conglomerados de 
polen hasta el estigma, añade también que estos insectos se alimentan de los tejidos de la flor en descomposición, de preferencia las lígulas, probablemente porque son la parte más carnosa de la flor. Estas particularidades pueden ocurrir en los diferentes sistemas de cultivo en $T$. cacao, debido a las condiciones ambientales cambiantes, lo cual puede interferir en el comportamiento de muchos insectos asociados a un cultivo específico (Soria, 1979).

Con respecto a la presencia de artrópodos en los diferentes sistemas de cultivo en $T$. cacao, Espinoza \& Ortiz (2011) manifiestan que los Hymenopteras (Formicidae), fueron el grupo más importante con un mayor número de ejemplares capturados. Similar a los resultados obtenidos a esta investigación, donde Hymenoptera fue el grupo de mayor cantidad de especies capturadas. Por otra parte, según Aguilar (1976), los insectos de la familia Formicidae se presentan una abundancia frecuente a escasa. A diferencia de los resultados de esta investigación que muestran en la mayoría de los clones que el mayor número de insectos capturados pertenecen a la familia Formicidae del orden Hymenoptera, considerándola con una abundancia muy frecuente o muy abundante.

Solano (2010) menciona que el mayor número de familias de insectos se encuentran en sistemas agroforestales y un menor número en sistemas de monocultivo, lo que no coincide con los resultados obtenidos en esta investigación, donde el mayor número de familias de insectos se registró en sistema de monocultivo y el menor para el sistema agroforestal. Según este autor, el índice de diversidad de Shannon, con respecto a las familias de insectos colectados, fue de 0.88 para el cacao en sistema agroforestal y 0.31 para el monocultivo.

\section{Conclusiones}

$\mathrm{S}_{\mathrm{i}}^{\mathrm{e}}$ determinó una baja diversidad y alta dominancia de insectos para los sistemas de producción analizados en la mayoría de los clones, a excepción del clon LR-35 donde se registró una mayor diversidad de insectos. La observación directa en las flores de T. cacao permitió identificar los insectos visitadores florales, siendo los dípteros de la familia Cecidomyiidae, los insectos polinizadores identificados, que inician su actividad entre las 9h00 - 11h00. Uno de los factores climáticos que influye en la presencia de los insectos en $T$. cacao es la humedad relativa, pues se encontró una fuerte relación de tendencia positiva entre estas dos variables.

\section{Literatura citada}

Adjaloo, M. K. y Oduro, W (2013). Insect Assemblage and the Pollination System of cacao (Theobroma cacao L).
Journal of Applied Biosciences, 13, 4588- 4594., Ghana.

Aguilar F (1976) Fauna desertica peruana. Invertebrados màs frecuentes en las lomas. Revista Peruana; volumen 1(19): p. 67-70.

Alvim, P. de T (1966). Factors affecting the flowering of cocoa tree. Cocoa Growers Bulletin 7:15-19.

Alvim P. de T (1984). Flowering of cocoa, Cocoa Growers' Bulletin, Vol. 35, pp. 23-31.

Amores, F., Agama, J., Suárez, C., Quiroz, J., y Motato, N. (2009). EET 575 y EET 576: Nuevos clones de cacao Nacional para la zona central de Manabí. Quevedo, Ecuador: INIAP, Estación Experimental Tropical Pichilingue. Boletín Divulgativo no. 346, pp 31., Estación Experimental Pichilingue., Ecuador.

Asomaning, E.J. A., Kwakwa, R.S. and Hutcheon, W.V. (1971). Physiological Studies on Amazon Shade and Fertilizer Trial at the Cocoa Research Institute. Ghana Journal of Agricultural Science, 4: 47-64., Ghana.

Bravo, J. Somarriba, E. Arteaga, G. (2011). Factores que afectan la abundancia de insectos polinizadores del cacao en sistemas agroforestales. Revista de Ciencias Agrícolas. Vol. 28, pp. 119-131., Costa Rica.

Córdoba C., Cerda R., Deheuvels O., Hidalgo E., Declerck F., (2013). Polinizadores, polinización y producción potencial de cacao en sistemas agroforestales de Bocas del toro, Panamá. Agroforestería en las Américas., $\mathrm{N}^{\circ}$. 49., Panamá.

De almeida, J. (2012). Respuestas ecofisiológicas de cacao (Theobroma cacao L.) a diferentes intensidades lumínicas. Tesis de grado. Universidad Cental de Venezuela., Venezuela.

Espinoza, G., Ortiz. (2011). Diversidad Entomologica de los insectos epigeos de las lomas cerro punta blancapachacamac, Lima. Durante el cambio de Estacion Otoño-Invierno 2011. Tesis de grado., Universidad Nacional Federico Villarreal, Perú.

Jaimez, R; Tezara, W; Coronel, I; Urich, R.(2008). Ecofisiología del cacao (Theobroma cacao): su manejo en el sistema agroforestal. Sugerencias para su mejoramiento en Venezuela. Revista Forestal Venezolana, 52(2), 253258., Venezuela.

Kaufmann, T. (1973). Preliminary observation on Cecidomyiid midge and its role as a cocoa pollinator in Ghana. Provisional communication., 6., pp. 193-198. Ghana.

Mavisoy, K., Cabezas, S., Ballesteros, W., \& Somarriba, E. (2009). Evaluación de la abundancia de Ceratopogonidae (Diptera) polinizadores de cacao (Theobroma cacao L.) en la hojarasca de 7 arboles de sombra. Tesis de grado., Universidad de Nariño., pp. 19., Costa Rica.

Mena-Montoya, Marlon, L. García Cruzatty, E. CuencaCuenca, L. Vera Pinargote, R. Villamar-Torres, S. Mehdi Jazayeri (2020). Flujo de polen de Theobroma cacao y su relación con factores climáticos en la zona central del Litoral Ecuatoriano. BIOAGRO, 32 (1): 39- 48. 
Mohr, H. and Schopfer, P. (1994). Plant Physiology. Springer Publishers., pp. 423 - 436. Alemania.

Noriega, A. (2012). Determinación de la habilidad combinatoria de 14 clones de cacao (Theobroma cacao L.) de tipo Nacional seleccionados. Santo DomingoEcuador: INIAP en la Estación Experimental Litoral Sur (EELS)., Ecuador.

Samsuri, T. (2013). The cacao flower visitor insects diversity and their potentialities as pollinators. . KnE Life Sciences, 4,540-543.

Solano, P. (2010). Diversidad de Insectos en Sistemas Agroforestalescon cacao en zona de influencia del cantón Quevedo., Los Ríos. Tesis de grado., Universidad Técnica Estatal de Quevedo., Ecuador.

Soria, Chapman, Knoke. (1981). Cacao pollination in Costa Rica: Breeding sites of Ceratopogonid (Diptera) midges. Revista Theobroma, Vol. 11(2), pp. 119-123.

Soria, S. (1979.). Insectos polinzadores: Forcipomyia metodos para aumentar la polinización y sus efectos sobre la produccion. $7^{a}$ conferencia internacional de pesquisas en cacau, $7^{a}$ conferencia internacional de pesquisas en cacau. Brasil. 